\title{
CQI Prediction for Shadow Fading in LTE-compatible GEO Mobile Satellite Communications System
}

\author{
Yadan Zheng, Mingke Dong, Ye Jin, Jianjun $\mathrm{Wu}^{\dagger}$ \\ Institution of Advanced Communications, EECS, Peking University \\ Beijing, 100871, China \\ E-mail: zhengyadan@pku.edu.cn, just@pku.edu.cn
}

\begin{abstract}
CQI(Channel Quality Indicator) is an essential indicator for AMC(Adaptive Modulation and Coding) technique in LTE. Due to the long delay of GEO satellite channel, CQI prediction is necessary to ensure effective AMC in LTE-compatible GEO mobile satellite communications system. The complete procedure and problem encountered when doing AMC in satellite system are introduced and the difficulties of prediction are analyzed. In order to obtain meaningful and feasible CQI prediction results, a complete prediction scheme is proposed. By using approximate CQI data which is smoothed and with only shadow fading influence, various prediction models are compared. Taking both computation complexity and prediction performance into account, two models, ARIMA (Autoregressive Integrated Moving Average) and Kalman filter are chosen to be applied in this scheme. Simulation results show that the prediction performance is very well with the proposed method, which can surely guarantee AMC performance.
\end{abstract}

Keywords-CQI; AMC; GEO; ARIMA; Kalman

\section{INTRODUCTION}

GEO mobile satellite system relies on high space satellites, thus making it very different from terrestrial system. It has the characteristics of wide downlink broadcast coverage, not sensitive to ground situations, wide band, good communication quality and also many other advantages.

AMC (Adaptive Modulation and Coding)[1,2] is utilized in many terrestrial systems, which is an essential method to improve system's capacity and data transmission efficiency. CQI (Channel Quality Indicator)[3] is calculated by UEs according to the downlink reference signals, and is sent back to eNodeB for AMC. But in satellite system, satellite channels' long propagation delay will cause trouble for AMC. So CQI prediction is of great needs to guarantee AMC performance.

Many existing prediction models are feasible for short-range prediction in terrestrial system, such as ARIMA (Autoregressive Integrated Moving Average)[4], long-range prediction proposed by Hallen[5], Kalman filter[6], etc. The prediction parameter includes CQI[4], Doppler shift[6], and channel matrix. All these models do prediction according to the input data sequence's autocorrelation. Their prediction ability are limited when doing long-range prediction much longer than the sequence' autocorrelation time. Therefore, in mobile satellite communication system, to guarantee AMC performance, the received CQI data's application method and the prediction model's selection are all necessary to take into consideration.

In Section II, GEO mobile satellite communication system and the procedure and problem of CQI transmission in AMC are introduced in details. Section III gives the concrete analysis for satellite channel quality, and various prediction models' comparison. In Section IV, the proposed prediction scheme is introduced, including the CQI data approximation and the model selection strategy. Section V shows the simulation result. A summary for this paper can be seen in Section VI.

\section{LTE-COMPATIBLE GEO MOBILE SATELLITE SYSTEM}

\section{A. Satellite Propagation Link Model}

In GEO mobile satellite communication systems, there exist two transmission modes, single-hop and double-hop mode. Typical application in satellite systems is double-hop mode, which will be the basic transmission model for this paper's analysis. The double-hop model comprises two signal paths: one is that signal transfers from the starting UE, is forwarded by a GEO satellite, and finally arrives at the destination ground station; and the other one is that signal transfers from the ground station, is forwarded by a GEO satellite similarly, and finally arrives at the destination UE. The complete process can be seen in Fig. 1. In GEO system, typical delay of double-hop mode is $540 \mathrm{~ms}$ [7]. This paper mainly focuses on the influence of the delay to AMC technique.

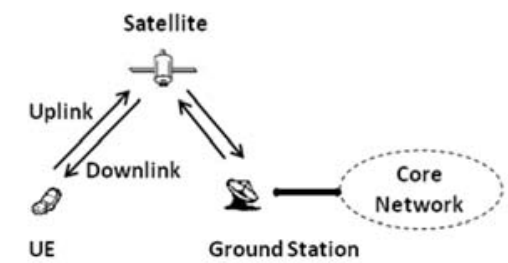

Figure 1. Double-hop model of satellite system

\section{B. Adaptive Modulation and Coding (AMC)}

Adaptive modulation and coding (AMC) is an advanced technique to improve data rate and make full use of channel capacity by applying different, adaptive order modulation schemes. It has been applied in many terrestrial systems. UE calculates CQI based on the downlink reference signals' SNR, and feed it back to eNodeB via uplink channel. After receiving CQI, eNodeB will allocate suitable downlink 
MCS based on the CQI and the resource distribution conditions. Overall, AMC is a strictly close loop process.

\section{AMC in GEO Satellite System}

In GEO mobile system, AMC is also needed to improve system performance. The mainly difference is the channel environment, including the transmission delay, Doppler frequency and so on.

To overcome the long delay problem, effective prediction is a sensible method to provide meaningful CQI data, but there is not much discussion about the CQI prediction in satellite environment so far. The challenge is that the channel variation situation and prediction model's characteristics must be considered jointly.

\section{SATElLite ChANNEL QuAlity MODEL AND PREDICTION MODEL}

\section{A. Satellite Channel Quality Model}

Models introduced by Loo[8], Lutz[9], and Corazza[10] are all commonly used satellite channel models. In this paper, a statistic model introduced in [11] is employed, which is a detailed satellite channel model based on Loo[8]. [11] used three-state model to describe direct signal: LOS conditions, moderate shadowing conditions, deep shadowing conditions. The transformation principle between these three states is a Markov chin. In each state, the detailed shadow fading situation varies under log-normal distribution, and has different distribution parameters. The small scale fading is under Rician distribution.

\section{B. Prediction Model}

One CQI data received by eNodeB can be received every TTI (1ms) when doing periodic feedback. Different prediction models may have different prediction ability. Here are some commonly used prediction models which are utilized in terrestrial system. The analysis focuses on the prediction performance, and calculation complexity.

1) Autoregressive Integrated Moving Average (ARIMA): ARIMA is a widely used channel quality prediction model. In terrestrial mobile system has already applied this model to predict CQI in 1TTI (1ms) range[4]. Here is definition of ARIMA (p,d,q) model:

$$
\hat{Z}_{t}(l)=E_{t}\left[\tilde{z}_{t+l}\right]=\sum_{j=1}^{l-1} \phi_{j} \hat{z}_{t}(l-j)+\sum_{j=l}^{p+d} \phi_{j} \tilde{z}_{t+l-j}-\sum_{j=l}^{q} \theta_{j} a_{t+l-j}
$$

Assume that received CQI state sequence is $z_{t}, z_{t-1}, z_{t-2} \ldots \hat{z}_{t}(l)$ is the minimum mean square error prediction for $l$ steps ahead of t. Assume $\mu$ as sequence's average value, and $a_{t}$ stands for the interference impulse. It has $\tilde{z}_{t}=z_{t}-\mu$. This model has a relative low computation complexity, and can output result in time.

Due to the heavy dependence on sequence autocorrelation, model prediction performance decreases quickly when the prediction range is longer than autocorrelation time. In this paper, the required prediction range is $540 \mathrm{~ms}$, as the $\mathrm{TTI}=1 \mathrm{~ms}$, 540 -step predictions are needed in this situation. According to Eq. (1), 540-step prediction will bring about huge computation quantity, also with much longer process delay. When the prediction range is long enough, the output will be the sequence's average value, which loses the value of prediction[12].

2) Long-range prediction [5]: Long-range prediction is firstly derived by Hellan. This method is based on AR model, and uses down sampled input data sequence as the prediction input. If the down-sample frequency fs satisfies Eq. (2), where fd is the maximum Doppler shift, the prediction can be done for a relative long-range with little information loss. The prediction range can be as long as several times of sequence autocorrelation time, up to around one wavelength[5].

$$
f s \geq 2 f d
$$

This method has a same computation complexity with ARIMA. But after down-sampling, the input data's interval becomes larger, for example, when sample interval is $20 \mathrm{~ms}$, the prediction step will be reduced to 27, which is much smaller than 540 steps for ARIMA model and the computation complexity decreased consequently. But in this paper, assume $\mathrm{S}$ band is utilized. $f_{c}=2.4 G H Z$, and $\lambda=c / f_{c}=0.125 \mathrm{~m}$, it is also very short compared with 540ms which makes difficulties for this method as well.

3) Kalman filter: Kalman filter is often used to do channel filtering, but actually, it is also an autoregressive prediction method[6]. Kalman filter is based on linear algebra and hidden Markov model. Future data state and value will be obtained gradually step by step. The model coefficients are calculated with the autocorrelation coefficients.

Kalman filter traces the hidden property of the input sequence and performs very well for predicting small scale fading in terrestrial system in short time distance. But the high computation complexity due to the matrix multiplication and the divergence problem when prediction range becomes longer are the limitation for Kalman filter.

\section{CQI PREDICTION SCHEME IN LTE-COMPATIBLE MOBILE SATELLITE COMMUNICATIONS SYSTEM}

\section{A. Data Approximation}

According to above, most of these prediction models' parameters and prediction abilities rely on input sequence autocorrelation. Even with Hallen's long-range method, only several times of autocorrelation's prediction can be achieved, which is far away from the required range. So the feasible and meaningful CQI prediction in GEO system cannot be achieved if using the models directly.

As the sequence's autocorrelation property can not satisfy the prediction models' requirement, approximation can be introduced in this situation. According to [11], satellite channel is influenced by both large scale and small scale fading. Large scale fading, which is mainly caused by shadow, varies much slower than small scale, which is caused by multipath and Doppler Effect. If smoothing small scale fading away, the left data sequence's autocorrelation time will increase significantly $[13,14]$. From the simulation 
result in [13], the channel quality with only large scale fading autocorrelation is much longer then that with both large and small scale fading, thus making it possible to meet the prediction models' requirement. What's more, since the data is smoothed, say averaged, for a period of time, the left data interval becomes longer, then the prediction step number reduces. The smooth method can also be various, such as moving average, exponential smoothing [15], etc. In this paper, moving average is applied to smooth away the small scale fading influence.

\section{B. Prediction Model Selection}

According to the analysis above, we can see that long-range prediction algorithm is basically an ARIMA model with less input data. While the approximate CQI data is already down-sampled by smoothing, this model is worthless in this situation; Finally, ARIMA and Kalman filter are left to be considered.

As said before, Kalman filter is a kind of autoregressive model as well, and the matrix's coefficients can be calculated using ARIMA model. Generally speaking, the prediction performance of ARIMA and Kalman filter can be almost the same in some situations.

As to the multi-step prediction, ARIMA needs to calculate data one step by one step, and when a new measured data arrives, the prediction has to be done again. On the other hand, Kalman filter needs to do matrix multiplication first and stores the result, and when a new measured data arrives, the prediction needs to do just one matrix multiplication. So actually, the computation complexity of these two models must be judged by concrete situations. When prediction step is small, the computation complexity can be viewed almost the same, but Kalman filter has a faster output as the coefficients are already stored. When prediction step is large, the matrix multiplication complexity cannot be endured anymore and the divergence cannot be neglected.

A prediction scheme is proposed as follows:

1) Approximation: Calculate the approximate CQI data using moving average or other smooth method.

2) Prediction model selection: When UE travels slowly (autocorrelation time much longer than prediction range), select low order Kalman filter or ARIMA model. When UE travels fast (autocorrelation time shorter than or same with prediction range), select high order ARIMA model.

3) Using approximate data to do prediction.

\section{V.SIMULATION AND ANALYSIS}

\section{A. Data Approximation Result}

The approximate data is compared with the original data in previous work [16]. The comparison shows that the difference is quite small that we can smooth away the small scale fading influence without much data loss. Meanwhile, the sequence's autocorrelation increases significantly in Fig. 2 , which greatly benefits the prediction. The proposed approximation method is feasible and meaningful to be applied in AMC process in satellite mobile system.

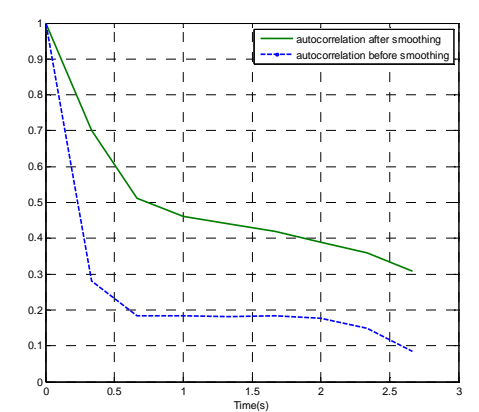

Figure 2. Comparison of correlation time between slow variation and fast variation CQI data

\section{B. Prediction Result with Approximate Data}

The prediction results are obtained with different parameters in Table I, including different UE speed. Since the prediction model has relationship with data autocorrelation time, the parameter UE speed is selected to ensure the data autocorrelation time is shorter than, almost equal to, longer than the prediction range. According to [14], we smoothed the data for every 2.5 meters.

TABLE I. SIMULATION PARAMETERS

\begin{tabular}{lll}
\hline Elevation $\mathbf{(}^{\mathbf{}}$ ) & UE Speed (m/s) & Prediction Range (ms) \\
\hline 80 & 10,50 & 500 \\
\hline
\end{tabular}

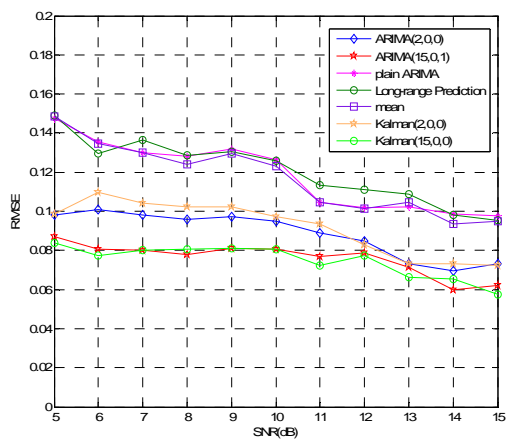

Figure 3. Prediction nerformance of different models

Fig. 3 gives the simulation comparison between low order ARIMA model, high order ARIMA model, low order Kalman Filter, high order Kalman filter using approximate data, and high order ARIMA model, long-range prediction, mean of data, using original data. The result shows that the RMSE of ARIMA model and long-range prediction with original data are the same with the RMSE of giving only mean of data. This means that predicting with original data is improper in this paper's situation. In the following, we use ARIMA model and Kalman filter to do predict. 


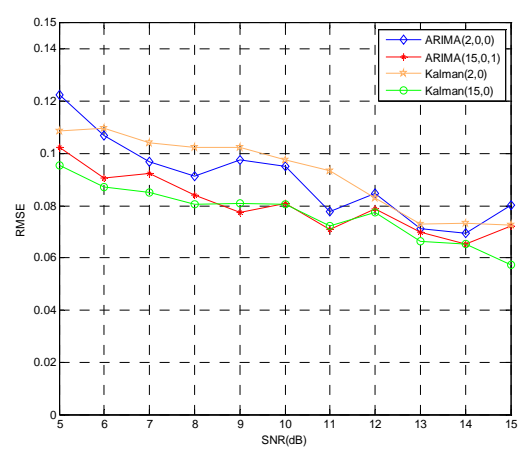

Figure 4. Prediction performance when UE travels at $10 \mathrm{~m} / \mathrm{s}$

Simulation is done for low-order ARIMA, high-order ARIMA, AR based low order Kalman filter, AR based high order Kalman filter for different parameters.

From Fig. 4, when UE travels slow, both ARIMA and Kalman filter of high order and low order perform almost the same with each other. As the prediction step is small, Kalman filter is enough to ensure the prediction performance with low complexity.

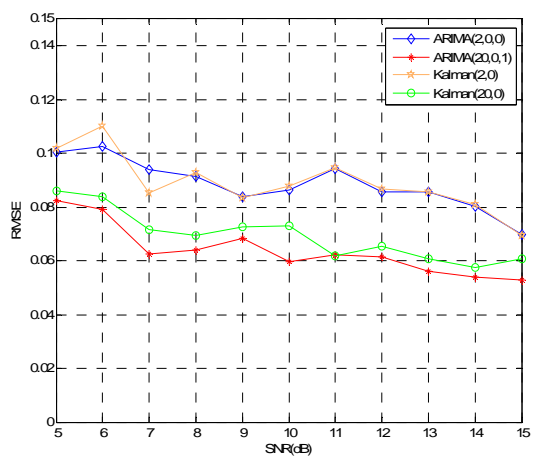

Figure 5. Prediction performance when UE travels at $50 \mathrm{~m} / \mathrm{s}$

When UE travels faster, as Fig. 5 shows, low order model becomes worse than high order model, and AR based Kalman filter performs worse than ARIMA.

\section{CONCLUSION}

The necessity and problem encountered of CQI prediction in AMC in LTE-compatible GEO mobile satellite communication system are discussed in this paper. In order to obtain feasible and rational prediction result to guarantee AMC performance, a complete prediction scheme is proposed in this paper by using approximate CQI data and proper prediction models, The approximation by smoothing small scale fading makes it possible to do prediction with existing prediction models. Comparisons of realization complexity and performance between various prediction models are provided as well. Simulation results show that the prediction performance is very well with the proposed method. A conclusion can be drawn that the proposed complete CQI prediction scheme can surely guarantee AMC performance.

\section{ACKNOWLEDGMENT}

This work is supported by the National High-Tech Research and Development Program of China (863 Program), No. 2012AA01A506. Corresponding author: Jianjun Wu; phone: +86-10-62752848; e-mail: just@pku.edu.cn.

\section{REFERENCE}

[1] 3GPP TS 36.213, "Evolved Universal Terrestrial Radio Access (E-UTRA); Physical layer procedures”, URL: www.3GPP.org.

[2] J. Shen, S. Suo, H. Quan, 3GPP Long Term Evolution: Principle and System Design, P eople Post Press, Beijing, 2008, Chaps5.4.3.

[3] S. Sesia, I. Toufik, M. Baker, LTE-UMTS Long Term Evolution From Theory to Practice, Chinese version, People Post Press, 2009, pp. 160-165.

[4] Y. Shang, X. Chen, "Channel Quality Indicator Prediction and Compensation Method and System”, Patent No. CN101958765A, Jan. 2011.

[5] Duel-Hallen, A., Hu, S., Hallen, H. "Long-range prediction of fading signals”, Signal Processing Magazine, IEEE, Vol. 17 , May 2000, pp.62-75.

[6] A. A. Heidari1, K. Khandani, D. McAvoy, "Adaptive modelling and long-range prediction of mobile fading channels", IET Commun., 2010, Vol. 4, Iss. 1, pp. 39-50

[7] K. Wang, Z. Gao, J. Du, J. Guo, Y. Cheng, J. Wu, “A Handoff Method for single Hop Satellite Mobile Communication System”, Sixth Annual Conference of the satellite communications new business and new technology, Beijing, Mar. 2010, pp382-389.

[8] C. Loo, “A Statistical Model for a Land Mobile Satellite Link”, IEEE Transactioks On Vehicular Technology, Vol. VT-34, No. 3, Aug. 1985, pp. 122-127.

[9] Lutz, D. Cygan, M. Dippold, F. Dolainsky, W. Papke, "The Land Mobile Satellite Communication Channel-Recording, Statistics, and Channel Model”, IEEE Transactions On Vehicular Technology, Vol. 40. No. 2, May 1991, pp. 375-386.

[10] G. E. Corazza, F. Vatalaro, "A statistical model for land mobile satellite channels and its application to nonstationary orbit systems," IEEE Transactions on Vehicular Technology, Vol. 43, No. 3, Aug. 1994, pp. 738-742.

[11] F.P. Fontan, M. Vazquez-Castro; C.E. Cabado, J.P. Garcia, E. Kubista, "Statistical Modeling of the LMS Channel" IEEE Transactions On Vehicular Technology, Vol. 50, No. 6, Nov. 2001 pp. 1549-1567.

[12] H. Cheng, P. N. Tan, J. Gao, J. Scripps, "Multi Step ahead Time Series Prediction”, In Proc. of the Pacific-Asia Conf on Knowledge Discovery and Data Mining, Vol. 3918, 2006, pp765-774.

[13] F. Fontan, M.A. Vazquez-Castro, "S-band LMS propagation channel behaviour for different environments, degrees of shadowing and elevation angles”, IEEE Transactions On Broadcasting, Vol. 44, No. 1, Mar. 1998, pp.40-76.

[14] P. Taaghol, R. Tafazolli, "Correlation model for shadow fading in land mobile satellite systems”, ELECTRONICS LETTERS, $17^{\text {th }}$, Vol. 33, No. 15, Jul. 1997, pp. 1287-1289.

[15] Brown, R. G., Meyer, R. F., "The fundamental theorem of exponential smoothing," Operations Research, Vol. 9, No. 5, Sep. Oct., 1961, pp. 673-687.

[16] Y. Zheng, S. Ren, X. Xu, Y. Si, M. Dong, J. Wu, "Channel Quality Approximation Using Large Scale Model in GEO Mobile Satellite System", The International Workshop on Electromagnetism and Communication Engineering, Neimenggu, Mar. 2012 